\title{
Diarréia aguda grave associada à Escherichia coli enteropatogênica clássica (EPEC): características clínicas e perdas fecais em lactentes hospitalizados
}

\author{
C.A.G. Oliva, I. Scaletsky, M.B. de Morais, U. Fagundes Neto \\ Disciplinas de Gastroenterologia Pediátrica e Microbiologia da Universidade Federal de São Paulo - Escola Paulista de M edicina, São \\ Paulo, SP.
}

RESUMO - Escherichia coli enteropatogênica clássica (EPEC) tem sido o agente enteropatogênico mais freqüentemente isolado nas fezes de lactentes, de baixo nível socioeconômico, hospitalizados com diarréia aguda grave, na cidade de São Paulo.

Oвj etıvos. 1) Analisar as características clínicas de lactentes do sexo masculino, hospitalizados com diarréia aguda grave associada à EPEC; 2) quantificar suas perdas fluidas fecais, ingestão de fór mulas alimentares e variações de peso corporal durante a evolução intra-hospitalar.

Casuística e Métodos. E studaram-se 38 lactentes do sexo masculino. Foram avaliadas as características clínicas e epidemiológicas dos pacientes e a freqüência dos diferentes sorogrupos de EPEC identificados. As crianças foram mantidas em cama metabólica, e balanços metabólicos diários foram obtidos durante toda a duração do quadro diarréico. Os lactentes permaneceram, em média, 5,8 dias em estudo metabólico. Duzentos e vinte balanços diários foram acumulados e analisados.

Resultados. $O$ grupo estudado apresentou como principais características clínicas: idade inferior a 12 meses, peso insuficiente ao nascer; desmame precoce e formas graves de desnutrição protéicocalórica. 0 sorogrupo 0111 foi o mais freqüentemente identificado (68,4\%), sendo a idade dos pacientes com tal agente significativamente maior que a dos portadores do sorogrupo 055.

A média dos volumes fecais diários foi de $66 \mathrm{~mL} /$ kg/dia, havendo diferenças segundo dias de estu-

\section{INTRODUÇÃO}

Diarréia aguda é uma síndrome prevalente na infância e observada com mai or freqüência nas crianças de tenra idade ${ }^{1,2}$. O impacto dessa síndrome na morbidade é tanto maior quanto menor a idade das crianças acometidas, seja no número de episódios por criança por ano, seja nas taxas de hospitalização $0^{3-7}$. Diversos estudos realizados em centros urbanos de países em desenvol vimento têm mostra- do. A média de ingestão diária de fórmulas alimentares foi de $85,2 \mathrm{~mL} / \mathrm{kg} / \mathrm{dia}$. Leite de vaca foi a dieta mais precocemente utilizada e as crianças que dela fizeram uso apresentaram volumes fecais maiores que os que receberam caseinato ou nutrição parenteral e incremento de peso corporal menor que os que utilizaram hidrolisado protéico OU NPT.

Conclusões. Os sorogrupos de EPEC acarretaram perdas fluidas fecais de moderada e grave intensidade nos lactentes estudados. A duração da diarréia mostrou curso agudo (menor ou igual a 14 dias) na maioria das crianças estudadas, reafirmando a evolução potencialmente autolimitada da diarréia aguda. Sete $(18,4 \%)$ pacientes, entretanto, desenvolveram quadro de diarréia persistente, indicando que tal evolução é esperada em uma parcela razoável de lactentes jovens hospitalizados com tal enfermidade. Os volumes médios de ingestão das fórmulas alimentares encontrados mostram o impacto da diarréia aguda na diminuição da ingestão alimentar. As médias observadas nas três diferentes fórmulas alimentares ficaram abaixo da ingestão calórica sugerida como padrão pela FAO/WHO (100cal/kg/dia). Não houve diferenças entre os volumes de aceitação segundo os tipos de fórmulas alimentares utilizadas.

UNITERMOS: Diarréia aguda. Escherichia coli enteropatogênica (EPEC). Balanço metabólico. Cama metabólica. Perdas fluidas fecais.

do que os sorogrupos de Escherichia coli enteropatogênica clássica (EPEC) são os principais agentes enteropatogênicos em crianças menores de dois anos de idade pertencentes às classes socioeconômicas menos favorecidas, acometidas de formas graves de diarréia aguda9-11. No Brasil, e em São Paulo, EPEC tem sido apontada como o agente mais freqüentementeisol ado em crianças hospitalizadas, no primeiro ano de vida e, principalmente, nos primeiros seis meses de idade $\mathrm{i}^{12-15}$. 
Somente no início deste século é que começaram a ser conhecidas as perdas intestinais de água e eletrólitos nas fezes de crianças acometidas de síndromes diarréicas. Darrow ${ }^{8}$ apresentou os resultados de balanços de água e el etrólitos na recuperação de desidratação devida a quadros graves de diarréia aguda em crianças. As perdas hídricas desses pacientes atingiram médias diárias de $125 \mathrm{~g} / \mathrm{kg}$ de peso corporal. Esse estudo apontou, também, os déficits apresentados pelos pacientes nos balanços de sódio, cloro, potássio, fósforo e cálcio, lançando as bases para o desenvolvimento da terapêutica de reidratação oral e promovendo importante diminuição nas taxas de mortalidade por desidratação.

A importância dos sorogrupos de EPEC na epidemi ol ogia da diarréia aguda em lactentes jovens em nosso meio e a escassez de estudos sobre o comportamento clínico e das per das fecais, durante toda a vigência dessa enteroinfecção em crianças hospitalizadas com quadros graves, motivaramnos a desenvolver esta pesquisa, com os seguintes objetivos: 1) analisar as características clínicas de lactentes do sexo masculino; 2) quantificar as perdas fluidas fecais, ingestão de fórmulas alimentares e variações de peso corporal durante a evolução intra-hospitalar.

\section{CASUÍSTICA E MÉTOdOS}

No período de janeiro de 1986 a dezembro de 1992, 475 crianças, de ambos os sexos, foram internadas na Unidade Metabólica com diagnóstico de diarréia aguda e tiveram suas fezes col hidas para pesquisa de agentes enter opatogêni cos. Os seguintes agentes foram pesquisados: Escherichia coli enteropatogênica clássica (antígenos O e H), Escherichia coli enteroinvasora (EIEC), Escherichia coli enterotoxigênica LT, ST (ETEC), Shigella, Salmonella, Yersinia, Campyl obacter e Rotavi rus.

Em 134 (28,2\%) das crianças estudadas, al gum sorogrupo de EPEC foi identificado, isol adamente, à coprocultura. Dos 134 pacientes portadores de EPEC nas fezes, 87 (64,9\%) eram meninos. Destes, $38(43,7 \%)$ foram tratados em cama metabólica durante toda a duração da síndrome diarréica que motivou a hospitalização, constituindo-se no grupo de pacientes estudados, prospectivamente.

O presente estudo foi aprovado pela Comissão de Ética Médica do Hospital U mberto I e do Hospital São Paulo. Os pais ou responsáveis pel os pacientes firmaram consentimento escrito para partici pação das crianças no estudo.

Foram incluídas no estudo as crianças que preencheram os seguintes critérios: sexo masculino; hospitalização por diarréia aguda, com início dos sintomas conhecido pel os pais ou acompanhantes e duração igual ou inferior a dez dias; identificação de cultura pura para algum sorogrupo de EPEC nas fezes; permanência em cama metabólica até desaparecimento do sintoma diarréia. Foram excluídas do estudo as crianças com as seguintes características: desconhecimento preciso do início do quadro atual; presença, no momento da hospitalização, de outras infecções ou complicações extradigestivas; utilização de antibióticos na vigência do quadro atual; situações clínicas que impossibilitassem a permanência em cama metabólica.

Diarréia aguda foi definida como um processo sindrômi co de duração igual ou inferior a dez dias, de etiologia provavel mente infecciosa (viral, bacteriana ou parasitária), induzindo má-absorção de água e el etrólitos, gerando aumento do número de evacuações e do volume fluido fecal, acarretando à criança depl eção hidrossal ina de intensi dade variável ${ }^{1}$.

No momento da internação na U nidade M etabólica, os pacientes tiveram seu estado de hidratação classificado segundo critérios clíni $\cos ^{16}$ em hidratado; desidratado de I grau; desidratado de II grau ou desidratado de III grau. De acordo com a necessidade, as crianças foram reidratadas por via oral e/ou parenteral. U ma vez considerados hidratados, os pacientes for am col ocados em cama metaból ica e incluídos no estudo.

No momento imediatamente anterior ao ingresso no estudo e clinicamente hidratados, os pacientes foram pesados, sem roupas. Os val ores encontrados foram comparados com aquel es de referência para idade e sexo (National Center for Health Statistics - NCHS), permitindo o cálculo da relação de peso para idade. A relação de peso para a idade foi expressa em $z$-scores ${ }^{17}$ e os pacientes tiveram seu estado nutricional classificado pelos critérios de Gomez $^{18}$.

F oram obti dos os val ores do peso de nasci mento, em gramas. E ste dado se baseou na informação dos pais ou acompanhantes. A média do peso de nascimento dos pacientes foi comparada com a definida como padrão de referência para recém-nascidos brasileiros ${ }^{19}$.

Coincidentemente ao início do período de estudo em cama metabólica, os pacientes passaram a receber uma das dietas a seguir: fórmula láctea di luída ao terço ( $\mathrm{Ninho}^{\circledR}-10 \%$ com adição de sacarose na concentração de $5 \%$ ), descrita nos resultados como "leite de vaca"; fórmula contendo caseína, ácidos graxos de cadeia média e polímeros da glicose (AL $110^{\circ}$ ) em diluição integral, descrita nos resultados como "caseinato"; fórmula de hidrolisado de proteína de soro de leite ultrafiltrado integral 
(Alfaré ${ }^{\oplus}$ ), descrita nos resultados como "hidrolisado"; nutrição parenteral total (NPT) foi utilizada associadamente a jejum oral, em decorrência da falência do suporte nutricional oral (intolerância alimentar múltipla). A via de acesso preferencialmente utilizada foi a periférica.

As crianças foram colocadas em camas metabólicas, construídas segundo $\mathrm{F}$ omon ${ }^{20}$, visando coletar, em separado, as perdas fecais e urinárias. Foram realizados balanços hídricos diários. O volume fecal foi criteriosamente mensurado em interval os de oi to horas. Os pacientes permaneceram no estudo metabólico até deixar de apresentar fezes líquidas por, pelo menos, dois períodos consecutivos de oito horas. Os dados relativos aos volumes de perdas foram anotados a cada oito horas em ficha própria. Nessa ficha anotavam-se, ainda, os pesos corporais inicial, final e médio, volume de aceitação oral da dieta, tipo de dieta utilizada, além de outros dados de importância clínica. A cada 24 horas, esses dados foram transcritos para outra ficha de controle metabólico, sendo os dados desta última utilizados para os resultados deste trabalho. Visando conhecer a evolução da síndrome diarréi ca segundo o tempo de duração do sintoma e tempo de internação hospitalar dos pacientes, os balanços metabóli cos de 24 horas foram agrupados segundo o tempo, em dias completos, de permanência no estudo (tempo de estudo) e o tempo, em dias compl etos, de duração da síndrome diarréica (dias de diarréia).

Para a análise dos resultados utilizaram-se testes paramétricos e não-paramétricos, levando-se em consideração a natureza das distribuições e a variabilidade dos valores das variáveis estudadas. F oram aplicados os seguintes testes: a) teste $Z$ para comparação entre médias (utilizado para comparar a média do peso de nascimento do grupo estudado com a definida como padrão para o recém-nascido brasileiro); b) análise de variância por postos de Kruskall-Wallis (com o objetivo de comparar os volumes fecais diários, os volumes de aceitação da fórmula alimentar e as variações percentuais de peso corporal, segundo dias de estudo e dias de diarréia). Quando mostrou diferença significante, esta análise foi completada pelo teste de comparações múltiplas.

Em todos os testes, fixou-se em 0,05 ou $5 \%$ ( $p<$ 0,05) o nível para rejeição da hi pótese de nulidade.

\section{RESULTADOS}

A idade dos pacientes variou entre 1 e 15 meses, com mediana de quatro meses. Vinte e seis crianças $(68,4 \%)$ eram menores de seis meses e apenas um

\begin{tabular}{|lcr|}
\hline $\begin{array}{l}\text { Tabela 1 - Sorogrupos de EPEC identificados às coprocul- } \\
\text { turas dos pacientes }\end{array}$ \\
\hline Coproculturas & Número & $\%$ \\
E.coli O11 H- & 20 & 52,6 \\
E.coli O111 H4 & 6 & 15,8 \\
E.coli O119 H- & 7 & 18,4 \\
E.coli O55 H4 & 4 & 10,5 \\
E.coli O127 H- & 1 & 2,6 \\
\hline
\end{tabular}

paciente tinha mais de 12 meses de idade.

Todos os pacientes apresentavam diarréia como principal sintoma do quadro atual. Vômitos estavam presentes em 32 (84,2\%) crianças e febre em 25 $(65,8 \%)$ delas. A duração da síndrome diarréica dos pacientes, previamente ao ingresso no estudo, variou de 1 a 10 dias, com mediana de cinco dias .

Treze pacientes $(34,2 \%)$ vieram encaminhados para internação de outros hospitais do Sistema Único de Saúde e os restantes procuraram diretamente o Pronto Socorro do Hospital U mberto I. Todos apresentavam diagnóstico médico prévio de desidratação moderada ou grave e necessitaram de terapêutica de reidratação em serviço médico, indicando a hospitalização.

A média de peso de nascimento do grupo estudado foi de $2.937 \pm 601$ gramas, mostrando-se significativamente menor (teste $z$ - $x$ calculado $=3.1$, $z$ crítico $=1,96$ ) que a considerada como padrão de referência para o recém-nascido brasileiro(3.197 \pm $523 g)^{19}$.

Nenhum dos pacientes estudados vinha recebendo leite materno quando do início da doença diarréica atual e 15 já haviam sido hospitalizados, pelo menos uma vez, devido a síndromes diarréicas, excetuando-se a internação atual.

A mediana dos valores dos z-scores de peso para idade calculados para os pacientes, no momento do ingresso no estudo metabólico, foi de-1,73. Segundo critério de Gomez, 16 (42,2\%) pacientes apresentavam desnutrição de segundo ou terceiro graus.

Os diferentes sorogrupos de EPEC identificados nas coproculturas dos pacientes encontram-se na tabela 1.

A idade das crianças portadoras do sorogrupo O111 (média de 5,5 meses) foi, com significância estatística (análise de variância de KruskallWallis), maior que a daquelas que apresentavam coproculturas positivas para o sorogrupo 055 (média de 1,5 mês).

Os pacientes permaneceram em estudo metabólico, enquanto perduraram as perdas hídricas fecais. Esses períodos variaram de 1 a 13 dias. A mediana de permanência foi de cinco dias. Dos 38 pacientes, foram obtidos, assim, 220 balanços me- 
Tabela 2 - Tempo, em dias (d), de duração da diarréia (tempo de diarréia), em que as quatro diferentes dietas foram utilizadas pelas crianças hospitalizadas com diarréia aguda associada à EPEC

\begin{tabular}{lrcrc|}
\hline Dietas utilizadas & \multicolumn{4}{c|}{ Tempo (d) de diarréia } \\
\cline { 2 - 5 } & Média & DP & Moda & Mediana \\
Leite de vaca (LV) & 7,06 & 3,25 & 6,00 & 6,00 \\
Caseinato (CA) & 9,11 & 3,87 & 11,00 & 10,00 \\
Hidrolisado (HI) & 8,22 & 2,58 & 8,00 & 8,00 \\
NPT (NP) & 11,33 & 3,61 & 10,00 & 11,00 \\
\hline & & & \\
Análise de variância por postos de Kruskall-Wallis. \\
(LvxCAxHIxNP) \\
H calculado= 44,95*; H crítico= 7,82. \\
Teste de comparações múltiplas. \\
*NP > LV, CA e HI \\
* LV < CA e HI \\
\hline
\end{tabular}

tabólicos diários.

A distribuição do tempo, em dias, de duração da diarréia em que as quatro diferentes dietas foram utilizadas e suas diferenças encontram-se na tabeIa 2.

Os val ores dos volumes fecais diários (VFD) variaram de 11 a $219,6 \mathrm{~mL} / \mathrm{kg}$ de peso corporal, apresentando média de 66,1 e mediana de $60,1 \mathrm{~mL} / \mathrm{kg}$. Comparando-se os diferentes dias estudados, pudemos detectar que: OS VFD do dia 5 foram menores que os do dia 1; OS VFD do dia 8 foram menores que os dos dias 1 e 2; OS VFD do dia 9 foram menores que os dos dias 1, 2, 3, 4, 5 e 6; OS VFD do dia 12 foram menores que os dos dias 1, 2, 3, 4 e 6 (tabela 3).

Nenhum paciente foi estudado no primeiro dia do episódi o diarréico atual. Dezoi to $(47,4 \%)$ meninos mantiveram fezes diarréicas após o déci mo dia de doença e sete $(18,4 \%)$ del es após o décimo quinto dia de duração da enfermidade (tabela 4).

Os valores dos VFD, segundo os diferentes tipos de di etas utilizadas pel os pacientes durante o estudo, estão expressos na tabela 5 .

Os volumes diários de ingestão (VID) das fórmulas alimentares aferidos variaram de 6,4 a $294,8 \mathrm{~mL} / \mathrm{kg}$, com média de 85,2 e mediana de $74,5 \mathrm{~mL} / \mathrm{kg}$. Não foi possível detectar diferenças estatisticamente significantes nos valores dos VID das três diferentes fórmulas alimentares utilizadas pelos pacientes durante o estudo metabólico.

As variações percentuais diárias de peso corporal (VPP) dos pacientes oscilaram entre -9,4 e 6,9\%, com média de 0,15 e mediana de $0,43 \%$. As VPP das crianças variaram, significativamente, segundo os dias de estudo metabólico (tabela 6). Os pacientes que ingeriram leite de vaca apresentaram VPP significativamente menores que os que utilizaram hidrolisado protéico ou nutrição parenteral total (tabela 7).
Tabela 3 - Valores do volumes fecais diários, em mililitros por quilograma de peso corporal $(\mathrm{mL} / \mathrm{kg})$, das crianças hospitalizadas com diarréia aguda associada à EPEC, segundo tempo, em dias(d), de estudo metabólico (dias de estudo)

\begin{tabular}{|c|c|c|c|}
\hline \multirow[t]{2}{*}{ Dias de estudo (d) } & \multicolumn{3}{|c|}{ Volumes fecais diários $(\mathrm{mL} / \mathrm{kg})$} \\
\hline & Média & DP & Mediana \\
\hline 1 & 80,61 & 42,33 & 86,75 \\
\hline 2 & 74,62 & 38,68 & 64,40 \\
\hline 3 & 72,60 & 38,01 & 64,49 \\
\hline 4 & 67,61 & 33,88 & 65,65 \\
\hline 5 & 61,01 & 35,98 & 68,24 \\
\hline 6 & 59,54 & 28,98 & 48,10 \\
\hline 7 & 55,26 & 29,37 & 52,29 \\
\hline 8 & 46,80 & 18,61 & 52,50 \\
\hline 9 & 34,23 & 16,06 & 33,15 \\
\hline 10 & 45,58 & 12,14 & 48,50 \\
\hline 11 & 47,75 & 26,34 & 41,85 \\
\hline 12 & 26,33 & 7,81 & 28,40 \\
\hline 13 & 11,50 & 0,00 & 11,50 \\
\hline Total geral & 66,06 & 36,83 & 60,10 \\
\hline \multicolumn{4}{|c|}{$\begin{array}{l}\text { Análise de variância por postos de Kruskall-Wallis. } \\
\text { (dias } 1 \times 2 \times 3 \times 4 \times 5 \times 6 \times 7 \times 8 \times 9 \times 10 \times 11 \times 12 \times 13 \text { ) } \\
\text { H calculado }=29,30 * ; H \text { crítico= } 21,03 \\
\text { Teste de comparações múltiplas. } \\
{ }^{*} \text { dia } 5<\text { dia } 1 \\
\text { * dia } 8<\text { dias } 1 \text { e } 2 \\
\text { * dia } 9<\text { dias } 1,2,3,4,5 \text { e } 6 \\
\text { * dia } 12<\text { dias } 1,2,3,4 \text { e } 6 .\end{array}$} \\
\hline
\end{tabular}

Tabela 4 - Valores dos volumes fecais diários dos pacientes, em mililitros por quilograma $(\mathrm{mL} / \mathrm{kg})$ de peso corporal, segundo dias (d) de diarréia estudados

\begin{tabular}{cccc} 
Dias de estudo (d) & \multicolumn{3}{c}{ Volumes fecais diários(mL/kg) } \\
\cline { 2 - 4 } & Média & DP & Mediana \\
2 & 92,70 & 43,76 & 110,20 \\
3 & 86,78 & 44,19 & 87,90 \\
4 & 72,83 & 32,31 & 79,60 \\
5 & 83,28 & 57,79 & 81,20 \\
6 & 74,99 & 36,41 & 74,15 \\
7 & 79,54 & 40,64 & 68,38 \\
8 & 68,67 & 41,26 & 55,20 \\
9 & 64,36 & 34,35 & 58,00 \\
10 & 53,49 & 32,38 & 46,80 \\
11 & 63,07 & 27,44 & 62,45 \\
12 & 62,36 & 27,37 & 61,00 \\
13 & 51,44 & 26,99 & 51,00 \\
14 & 57,27 & 28,21 & 57,10 \\
15 & 57,64 & 19,36 & 53,58 \\
16 & 58,10 & 34,17 & 42,70 \\
17 & 40,53 & 14,28 & 36,50 \\
18 & 29,60 & 0,00 & 29,60 \\
19 & 25,40 & 0,00 & 25,40 \\
20 & 17,70 & 0,00 & 17,70 \\
21 & 11,50 & 11,50 & 0,00
\end{tabular}

Análise de variância por postos de Kruskall-Wallis

(dias $2 \times 3 \times 4 \times 5 \times 6 \times 7 \times 8 \times 9 \times 10 \times 11 \times 12 \times 13 \times 14 \times 15 \times 16 \times 17 \times 18 \times 19 \times 20 \times 21$ ) $\mathrm{H}$ calculado $=23,87 ; \mathrm{H}$ crítico $=30,14$ 


\begin{tabular}{|c|c|c|c|}
\hline \multirow[t]{2}{*}{ Dietas utilizadas } & \multicolumn{3}{|c|}{ Volumes fecais diários } \\
\hline & Média & DP & Mediana \\
\hline Leite de vaca (LV) & 83,20 & 41,11 & 87,50 \\
\hline Caseinato (CA) & 66,39 & 34,05 & 61,63 \\
\hline Hidrolisado (HI) & 71,37 & 40,79 & 66,95 \\
\hline NPT (NP) & 50,67 & 25,16 & 47,20 \\
\hline \multicolumn{4}{|c|}{$\begin{array}{l}\text { Análise de variância por postos de Kruskall-Wallis } \\
\text { H calculado }=22,85^{*} ; \mathrm{H} \text { crítico }=7,82 \text {. } \\
\text { Teste de comparações múltiplas } \\
{ }^{*} \mathrm{LV}>\mathrm{CA} \\
{ }^{*} \mathrm{NP}<\mathrm{LV}, \mathrm{CA} \text { e HI }\end{array}$} \\
\hline
\end{tabular}

\begin{tabular}{|c|c|c|c|}
\hline \multirow[t]{2}{*}{ Dias de estudo (d) } & \multicolumn{3}{|c|}{ Variação diária do peso corporal (\%) } \\
\hline & Média & DP & Mediana \\
\hline 1 & $-1,10$ & 2,67 & $-0,78$ \\
\hline 2 & 0,00 & 2,80 & 0,19 \\
\hline 3 & 0,17 & 2,24 & 0,55 \\
\hline 4 & 0,26 & 2,03 & 0,59 \\
\hline 5 & 0,48 & 1,80 & 0,26 \\
\hline 6 & $-0,23$ & 1,79 & 0,15 \\
\hline 7 & 1,67 & 2,00 & 0,99 \\
\hline 8 & 1,13 & 1,30 & 1,19 \\
\hline 9 & 1,10 & 2,46 & 0,72 \\
\hline 10 & 3,02 & 3,58 & 3,11 \\
\hline 11 & 0,24 & 2,09 & 0,51 \\
\hline 12 & 0,78 & 1,19 & 0,55 \\
\hline 13 & 0,00 & 0,00 & 0,00 \\
\hline Total geral & 0,15 & 2,39 & 0,43 \\
\hline \multicolumn{4}{|c|}{$\begin{array}{l}\text { Análise de variância por postos de Kruskall-Wallis } \\
\text { (dias } 1 \times 2 \times 3 \times 4 \times 5 \times 6 \times 7 \times 8 \times 9 \times 10 \times 11 \times 12 \times 13 \text { ) } \\
\mathrm{H} \text { calculado }=21,43^{*} ; \mathrm{H} \text { crítico= } 21,03 \text {. } \\
\text { Teste de comparações múltiplas } \\
\text { * dia } 1<\text { dias } 3,4,5,7,8,9 \text { e } 10 \\
\text { * dia } 6<\text { dia } 7\end{array}$} \\
\hline
\end{tabular}

\section{DISCUSSÃo}

Uma vez que o desenho do estudo pressupõe seguimento hospitalar, apenas foram sel ecionados pacientes com quadros graves que requereram internação. Assim, os resultados refletem o comportamento dos casos graves de diarréia aguda induzida por EPEC, não permitindo extrapolá-los para todo o espectro da doença. A necessidade de separar adequadamente as perdas fecais e urinárias tornou imperioso selecionar apenas os meninos para este estudo. Vários trabal hos em nosso meio mostram que a diarréia aguda associada à EPEC, em crianças hospitalizadas, afeta mais freqüentemente crianças do sexo masculino, sem mos-
Tabela 7 - Variações percentuais diárias do peso corporal das crianças hospitalizadas com diarréia aguda associada à EPEC, de acordo com os quatro diferentes tipos de dietas utilizadas

\begin{tabular}{|llcc|}
\hline Tipo de dieta & \multicolumn{3}{l}{ Variação do peso corporal diário (\%) } \\
\cline { 2 - 4 } \multicolumn{1}{c}{ utilizada } & Média & DP & Mediana \\
Leite de vaca (LV) & $-0,82$ & 2,22 & $-0,32$ \\
Caseinato (CA) & 0,04 & 3,01 & 0,40 \\
Hidrolisado (HI) & 0,18 & 2,01 & 0,57 \\
NPT (NP) & 0,84 & 2,12 & 0,78 \\
\hline NPT = nutrição parenteral total. & \\
Análise de variância por postos de Kruskall-Wallis \\
H calculado = 11,88 *; H crítico= 7,82. \\
Teste de comparações múltiplas \\
*LV < HI e NP \\
\hline
\end{tabular}

trar, entretanto, diferenças entre os sexos no tocante às complicações e/ou mortali dade ${ }^{21,22}$.

Resumidamente, podemos considerar que as crianças estudadas com diarréia aguda associada à EPEC apresentaram como características gerais a baixa idade, peso insuficiente ao nascer, desmame precoce e desnutrição protéico-calórica. Esse perfil vem sendo apontado por vários autores nos últimos $\operatorname{anos}^{23-25}$.

Pudemos observar que os pacientes portadores do sor ogrupo 055 tinham idade significativamente menor que aqueles em que se isolou o sorogrupo 0111. Alguns estudos têm mostrado o sorogrupo O55 relacionado a infecções em recém-nascidos, contaminados durante a permanência em unidades neonatais ${ }^{26,27}$.

A comparação das quatro di etas, segundo o tempo de estudo em que foram utilizadas, mostrou a seguinte ordem crescente de utilização: leite de vaca, caseinato, hidrolisado e nutrição parenteral total (NPT) associada a jejum oral. Quanto ao tempo de duração da doença diarréica em que as diferentes dietas foram utilizadas, pudemos detectar que a NPT foi mais tardiamente empregada que as fórmulas alimentares orais. Dentre estas últimas, leite de vaca foi utilizado em tempo anterior ao caseinato e hidrolisado protéico. Esses resultados refletem a prática atual do esquema de realimentação em lactentes hospitalizados com diarréia, em nosso meio.

Dos 38 pacientes estudados, $18(47,4 \%)$ mantinham diarréia após o décimo dia de doença e sete (18,4\%), após o décimo quarto dia. Esses achados reforçam a importância dos sorogrupos de EPEC como predisponentes de quadros diarréicos persistentes, já apontados na literatura ${ }^{28,29}$.

Os volumes fecais diários (VFD) variaram de 11 a $219,6 \mathrm{~mL} / \mathrm{kg}$, com mediana de $60,1 \mathrm{~mL} / \mathrm{kg}$. Esses achados reforçam a importância da EPEC como causadora de casos graves de diarréia aguda em 
lactentes jovens, com grandes perdas hídricas e el etrolíticas fecai $\mathrm{s}^{30}$. Quando anal isamos o comportamento doS VFD segundo os dias de estudo metabólico, pudemos detectar diferenças estatisticamente significantes, com médias maiores nos primeiros dias de estudo. Os VFD mostraram diferenças segundo o tipo de dieta utilizada pelos pacientes. Os lactentes em NPT e jejum oral apresentaram VFD menores que os realimentados com fórmulas alimentares orais, fato este já há muito conhecido ${ }^{31,32}$. As crianças real imentadas com leite de vaca mostraram VFD maiores que aquel as que utilizaram caseinato. Cabe a ressalva que as dietas foram empregadas em tempos diferentes de estudo e de diarréia, não sendo possível afirmar que as variações doS VFD observadas decorreram exclusivamente do tipo de dieta utilizada. Palma ${ }^{30}$ observou que lactentes portadores de sorogrupos de EPEC e realimentados com fórmulas contendo lactose apresentavam VFD maiores que os realimentados com fórmulas isentas desse carboidrato, nas primeiras 48 horas de hospitalização.

A aceitação oral de fórmulas alimentares variou de 6,4 a $294,8 \mathrm{~mL} / \mathrm{kg} /$ dia nas crianças estudadas. A mediana dos volumes ingeridos foi de $74,5 \mathrm{~mL} / \mathrm{kg}$, denotando a anorexia freqüente nesses quadros.

As variações percentuais diárias do peso corporal (VPP) das crianças estudadas oscilaram entre $-9,48$ e $6,92 \%$, com mediana de $0,43 \%$. As VPP no primeiro dia de estudo foram maiores que as verificadas nos dias 3, 4, 5, 7, 8, 9 e 10. Tal observação deve ser fruto da mai or dificuldade em repor as perdas hidroeletrolíticas no primeiro dia de estudo, pois não se conheciam, objetivamente, as perdas reais dos pacientes. Os pacientes real imentados com lei te de vaca apresentaram VPP menores que os que utilizaram hidrolisado protéico ou NPT.

Em nossa investigação, a utilização da cama metabólica permitiu avaliar, de modo simples e preciso, as principais características do quadro diarréi co, facilitando a reposição das perdas hídricas e cor reção dos distúr bi os el etrol íti cos em bases menos empíricas e de modo mais lógico e científico, trazendo importantes informações sobre a história natural da enteroinfecção grave induzida por EPEC em lactentes jovens hospitalizados.

Este trabalho foi realizado com auxílio financeiro do CNPq.

\section{SUMMARY}

Severe acute diarrhea associated to classic enteropathogenic by clinical features and fecal losses in hospitalized infants
Enteropathogenic Escherichia coli (EPEC) strains are the most prevalent enteropathogenic agents isolated in the stools of hospitalized infants with severe acute diarrhea in São Paulo. These mi croorganisms induce a severe intestinal secretion of fluids and electrolytes that can cause dehydration leading to hospital admission in the majority of the cases.

OвJECTIVES. This investigation aims at the following objectives: 1 ) to study the clinical features of acute diarrhea in male infants who were hospitalized owing to EPEC infection; 2) to determine the fecal fluid losses, formula intake and variation of thebody weight during theevolution of the disease.

Patients and Methods. Thirty eight maleinfants with acute diarrhea due to EPEC strains were studied. The clinical and epidemiological features of the patients were recorded and the different EPEC serogroups were identified. The infants were kept in a metabol ic bed in order to all ow the coll ection of stools and urineseparatel y during the whol eperiod of the disease, and daily metabolic balances were also obtained. The mean duration of the metabolic study was 5.8 days, and during this period of time 220 daily metabolic balances were accumulated and analyzed.

RESULTS. The main clinical features of the studied group wereas foll ows: age bel ow 12 months; I ow birth weight; precocious weaning; severe proteincalorie malnutrition. EPEC O111 was the most frequent serogroup identified, present in $68.4 \%$ of the infants. Theaverage daily fecal fluid losses were 66 $\mathrm{ml} / \mathrm{kg}$ and the mean daily formula intake was 85.2 $\mathrm{ml} / \mathrm{kg}$. Cow's milk was the most precocious food utilized and the infants who received cow's milk feeding presented the largest stool losses in comparison with lactose free formulas and total parenteral nutrition.

CONCLUSION. EPEC strains are able to induce moderate to severe fecal fluid losses in infants and the duration of diarrhea is usually bel ow 15 days, although in several circumstances diarrhea can show a protracted evolution owing to food intolerance associated or not with intestinal secretion. [Rev Ass Med Brasil 1997; 43(4): 283-9.]

KEY WORDS: Acute diarrhea. Enteropathogenic Escherichia coli (EPEC). Metabolic balance. Metabolic bed. Fecal fluid losses.

\section{REFERÊ NCIAS BIBLIOGRÁFICAS}

1. Fagundes Neto U, Wehba J, Penna FJ. Gastroenterologia pediátrica. 2a ed. Rio de J aneiro, Medsi, 1991.

2. Snyder J D, Merson MH. The magnitude of the global problem of acute diarrhoel disease: a review of active 
surveillance data. Bull WHO 1982; 60: 605-13.

3. Ho MS, Glass RI, Pinsky PF et al. Diarrheal deaths in American children: are they preventable? J AMA 1988; 260: 3.281-5.

4. Black RE, Brown $\mathrm{KH}$, Becker S. Longitudinal studies of infectious diseases and physical growth of children in rural Bangladesh. Am J Epidemiol 1982; 115: 315-24.

5. Figueroa G, Araya M, I banez S. Enteropathogens associated with acute diarrhea in hospitalized infants. J Pediatr Gastroenterol Nutr 1986; 5(2): 226-31.

6. Flores J, Nakagomi $\mathrm{O}, \mathrm{Nakagomi} \mathrm{T}$. The role of rotaviruses in pediatric diarrhea. Pediatric Infec Dis 1986 5(1): S53-62.

7. Fundo das Nações Unidas para a Infância (UNICEF). Situação Mundial da Infância. Brasília, UNICEF, 1991.

8. Darrow DC. The retention of electrolyte during recovery from severe dehydratation due to diarrhea. J Pediatr 1946; 28: $515-40$.

9. Black RE, Lopes de Romana G, Brown KH. Incidence and etiology of infantile diarrhea and major routes of transmission in Huascar, Peru. Am J Epidemiol 1989; 129(4):785-99.

10. Fagundes Neto U, Reis J C, Sabbag NA, Oliva CAG. Diarréia aguda: evolução clínica em crianças. Rev Paul Pediatr 1989; 7: $27-34$

11. Fagundes Neto U, Oliva CAG, Gallo $P$ et al. Mortalidade infantil por diarréia. Rev Paul Pediatr 1991; 9: 101-11.

12. Magalhães $M$, Andrade $M$, Carvalho AE. Pathogenic E scherichia coli associated with infantile diarrhea. Rev Microbiol 1981; 12: 38-41.

13. Toledo MRF, Alvariza MCB, Murahovschi J, Ramos SRTS, Trabulsi LR. Enteropathogenic E. coli serotypes and endemic diarrhea in infants. I nfect I mmun 1983; 39: 586-9.

14. Levine MM, Edelman R. Enteropathogenic Escherichia coli of classic serotypes associated with infant diarrhea: epidemiology and pathogenesis. Epidemiol Rev 1984, 6:31-51.

15. Baldini MM, Kaper JJ , Levine MM, Candy DCA, Moon HW. Plasmid-mediated adhesion in enteropathogenic Escherichia coli. J Pediatr Gastroenterol Nutr 1983; 2: 534-8.

16. Marcondes E. Desidratação e desnutrição em pediatria. São Paulo, Sarvier, 2a ed, 1971.

17. Organizacion Mundial de la Salud (OMS). Medicíon de cambio del estado nutricional. Ginebra, 1983.

18. Goméz F, Ramos RG, Frenk $S$ et al. Mortality in second and third degree malnutrition. J Trop Pediatr 1956; 2: 77-83.

19. Nóbrega FJ (coord). Antropometria, patologias e malforma- ções congênitas do recém-nascido brasileiro e estudos de associação com al gumas variáveis maternas. J Pediatr 1985; 59(supl 1): 6-113.

20. Fomon SJ . Infant nutrition. $2^{\text {nd }}$ ed. Philadelphia, Saunders, 1974.

21. Fagundes-Neto U, Patricio FRS, Wehba J et al. An Escherichia coli strain that causes diarrhea by invasion of the small intestinal mucosa and induces monossacharide intolerance. Arq Gastroenterol 1979 16:205-8.

22. Lifshitz F, Fagundes Neto U, Oliva CAG, Cordano A, Friedman S. Refeeding of infants with acute diarrheal disease. J Pediatr 1991; 118: S99-110.

23. Kitagawa SMJ , Toledo MRF, Trabulsi LR et al. Etiologia da diarréia infecciosa endêmica: da criança de baixo nível socioeconômico em São Paulo. Rev Paul Pediatr 1989, 7: 16-25.

24. Andrade J RC, Silva CC. Endocytosis-inducer adhesins produced by enteropathogenic serogroups of Escherichia coli participate on bacterial attachment to infant enterocytes. Mem Inst Oswaldo Cruz 1987; 82(1): 87-90.

25. Silva GAP. Diarréia aguda associada a Escherichia coli enteropatogênica clássica em lactentes de baixas condições soci oecômicas na ci dade do Recife. Estudo clínico e mi crobiológico.1991, São Paulo. [Tese de doutorado apres. à Escola Paulista de Medicina.]

26. Prado U, Braun S, Bosch P. Análise de Escherichia coli enteropatogênica clássica como causa endêmica de diarréia aguda em crianças chilenas. Rev Chil Pediatr 1984; 55(3): 171-5.

27. Donnenberg MS, Donohue-Rolfe A, Keusch GT. Epitelial cell invasion: an overlooked property of enteropathogenic Escherichia coli (EPEC) associated with the EPEC adherence factor. J Inf Dis 1989; 160: 452-9.

28. Brasil. Ministério da Saúde. Secretaria de Assistência à Saúde. Coordenação de Saúde Materno-I nfantil. Assistência e Controle das Doenças Diarréicas.1993; Brasília, Centro de Documentação do Ministério da Saúde

29. American Academy of Pediatrics. Committee on Nutrition. Use of oral fluid therapy and post-treatment feeding following enteritis in children in a developed country. Pediatrics 1985; 75: 358-61.

30. Palma D. Diarréia aguda: perdas hídricas fecais em lactentes hospitalizados e sua correlação com a presença de lactose na dieta. 1992. São Paulo. [Tese de mestrado apres. à Escola Paulista de Medicina.] 